\title{
Optimal control of autonomous linear systems switched with a pre-assigned finite sequence
}

\author{
Alessandro Giua (*), Carla Seatzu (*), Cornelis Van Der Mee (**) \\ (*) University of Cagliari, Department of Electrical and Electronic Engineering \\ Piazza d'Armi - 09123 Cagliari, Italy \\ \{giua, seatzu\}ediee.unica.it \\ http://www.diee.unica.it/ giua/info.html \\ http://www.diee.unica.it/ seatzu/info.html \\ (**) University of Cagliari, Department of Mathematics \\ Via Ospedale, 72 - 09100 Cagliari, Italy \\ cornelis@krein.unica.it \\ http: //riemann.unica.it/ cornelis/
}

\begin{abstract}
The paper deals with the optimal control of switched piecewise linear autonomous systems, where the objective is that of minimizing a performance index over an infinite time horizon. We assume that the switching sequence has a finite length and is pre-assigned, while the unknown switching times are the optimization parameters. We also assume that at each switch a jump in the state space may occur and that a cost may be associated to each switch.

The optimal control for this class of systems takes the form of a state feedback, i.e., it is possible to identify a region of the state space such that an optimal switch should occur if and only if the present state belongs to this region. We show how such a region can be computed with a numerical procedure and show that, in the particular case in which the switching costs is null, the region is homogeneous.
\end{abstract}

\section{INTRODUCTION}

Switched systems are a particular class of hybrid systems consisting of a number of subsystems (that may also be infinite) and a switching law that indicates the active subsystem at each time instant. Examples of switched systems may be found in many application fields, such as chemical processes, transportation systems, electrical circuits, and so on.

The problem of determining optimal control laws for this class of hybrid systems has been widely investigated in the last years and many results can be found in the control and computer science literature [2], [4], [5], [7], [11]. Many of these works propose control procedures that are based on the discretization of state space into grids and use search methods to find optimal open-loop solutions. Approaches of this kind may often reveal difficult to apply in real cases because of the computational complexity and may also result to be not accurate enough. We also mention the contribution given by Riedinger et al. in [7], [8], [9] where very general sufficient conditions for optimal control problems of switched systems are given in terms of hamiltonian function.

In this paper we restrict our attention to the case of switched systems whose subsystems are linear and autonomous. We also assume that the switching sequence is finite and pre-assigned. Thus, our problem is that of determining the optimal switching times $\tau_{j}, j=1, \cdots, n$, at which the hybrid system switches be- tween autonomous linear dynamics of the type $\dot{\boldsymbol{x}}=\boldsymbol{A}_{j} \boldsymbol{x}$, where the sequence $\boldsymbol{A}_{j}, j=1, \cdots, n$ is known. We also generalize this framework by assuming that whenever at time $\tau_{j}$ a switch from $\boldsymbol{A}_{j}$ to $\boldsymbol{A}_{j+1}$ occurs, the state should jump from $\boldsymbol{x}\left(\tau_{j}^{-}\right)$to $\boldsymbol{x}\left(\tau_{j}^{+}\right)=\boldsymbol{M}_{j} \boldsymbol{x}\left(\tau_{j}^{-}\right)$.

In general, assume that the initial and final times are $\tau_{0}=0$ and $\tau_{n+1}=\infty$ and that $k$ out of the $n$ allowed switches occur (i.e., occur in a finite amount of time). Given a choice of switching times

$$
0=\tau_{0} \leq \tau_{1} \leq \cdots \leq \tau_{k}<\tau_{k+1}=\cdots=\tau_{n}=\tau_{n+1}=+\infty
$$

we consider a performance index of the form:

$$
F\left(\tau_{1}, \cdots, \tau_{n}\right)=\int_{0}^{\infty} \boldsymbol{x}^{T}(t) \boldsymbol{Q} \boldsymbol{x}(t) d t+\sum_{j=1}^{k} H_{j},
$$

i.e., the performance index is composed of a cost associated to the continuous evolution and of a cost associated to the switches. In particular,

- $\boldsymbol{Q}$ is a positive definite matrix that weights the continuous state;

- $H_{j}$ is the cost of the $j$-th switch.

The control problem we investigate consists in determining the optimal switching times so as to minimize the performance in$\operatorname{dex} F$.

We make the following assumptions:

1. Each matrix $\boldsymbol{A}_{j}$ is stable, thus the switched system is stable regardless of the choice of switching times for any finite $n$.

2. Each system is autonomous, i.e., we do not need to compute a continuous control. The only control input for this system is the controlled switch/jump. Also switch and jump are coupled, in the sense that the $j$-th jump and the $j$-th switch are triggered by the same event and occur at the same time.

3. We assume that a finite constant cost is associated to a each switch that occur in a finite time. Saying that only $k$ out of the $n$ switches occur in finite time is equivalent to saying that only the first $k$ out of the $n$ allowed switches occur: i.e., only a prefix of the switching sequence may be executed if convenient. 
The results we present show that the optimal control law turns out to be a "state-feedback", in the sense that for all $j \leq n$ it is possible to identify a region $\mathcal{C}_{j, n}$ of the state space such that the $j$-th switch should occur if and only if we are within this region. Furthermore if the switching costs are all null, this region is homogeneous, i.e., if $\boldsymbol{x} \in \mathcal{C}_{j, n}$ then $\lambda \boldsymbol{x} \in \mathcal{C}_{j, n}$, for all real numbers $\lambda$.

The original features of our approach can be summarized as follows. Firstly, our derivation is based on the analytical derivation of the cost functional rather than the hamiltonian. Secondly, we are able to show that in this particular case the optimal control is a state feedback (and not an open-loop control). Thirdly, we are able to compute with a simple numerical procedure not only necessary but also sufficient conditions for optimality.

One limitation of the present approach is the fact that the switching sequence is pre-assigned. In effect, preliminary results that are not discussed here, show that our approach can easily be generalized to consider a (possibly infinite) set of legal sequences provided that they can all be generated by a finite state automaton over the alphabet $\mathcal{A}$. We observe, however, that there exist significant problems of practical relevance where the present framework (pre-assigned sequence) may be successfully applied. Consider, as an example, an active filtering problem where by connecting or disconnecting a capacitor one aims to reduce the distortion of an output signal. Such a problem can be framed as a pre-assigned sequence of switches $\boldsymbol{A} \rightarrow \tilde{\boldsymbol{A}} \rightarrow \boldsymbol{A} \rightarrow \cdots$, where $\dot{\boldsymbol{x}}(t)=\boldsymbol{A} \boldsymbol{x}(t)$ is the dynamics of the system with the capacitor connected and $\dot{\boldsymbol{x}}(t)=\tilde{\boldsymbol{A}} \boldsymbol{x}(t)$ is the dynamics of the system with the capacitor disconnected.

It may be possible to extend the results we present here to the cases - considered in the literature already mentioned where the subsystem dynamics are not all stable but there exists a stabilizing switching sequence; this is a topic for future work.

The paper is structured as follows. In Section 2 we state the class of systems considered and the optimization problem we want to solve. In Section 3 we show that when a finite number of switches are allowed, the optimal control is a feedback law and we present a constructive technique to determine the switching regions. In Section 4 a complete example is discussed.

\section{The System With Switching CONDITIONS}

\section{A. System Dynamics}

Given the switching times $0=\tau_{0} \leq \tau_{1} \leq \cdots \leq \tau_{n} \leq$ $\tau_{n+1}=\infty$, the $N \times N$ stable matrices $\boldsymbol{A}_{1}, \cdots, \boldsymbol{A}_{n+1} \in \mathcal{A}$ and the switching matrices $\boldsymbol{M}_{1}, \cdots, \boldsymbol{M}_{n}$, consider the linear system whose dynamics are given by

$$
\left\{\begin{array}{l}
\dot{\boldsymbol{x}}(t)=\boldsymbol{A}_{j} \boldsymbol{x}(t), \\
\text { for } \tau_{j-1}<t<\tau_{j}, \\
\boldsymbol{x}\left(\tau_{k}^{+}\right)=\boldsymbol{M}_{k} \boldsymbol{M}_{k-1} \cdots \boldsymbol{M}_{j} \boldsymbol{x}\left(\tau_{j}^{-}\right), \\
\quad \text { for } \tau_{j-1}<\tau_{j}=\cdots=\tau_{k}<\tau_{k+1}, \\
\boldsymbol{x}(0)=\boldsymbol{x}_{0} .
\end{array}\right.
$$

Then we define the evolution matrices $\boldsymbol{U}(t, \tau)(t>\tau \geq 0)$ by

$$
\boldsymbol{x}\left(t^{-}\right)=\boldsymbol{U}(t, \tau) \boldsymbol{x}\left(\tau^{+}\right) .
$$

Then, obviously, for $\tau_{j-1}<\tau_{j}=\cdots=\tau_{k}<\tau_{k+1}$,

$$
\boldsymbol{U}\left(\tau_{k}^{+}, \tau\right)=\boldsymbol{M}_{k} \boldsymbol{M}_{k-1} \cdots \boldsymbol{M}_{j} \boldsymbol{U}\left(\tau_{j}, \tau\right),
$$

and

$$
\boldsymbol{U}\left(t, \tau_{j}^{-}\right)=\boldsymbol{U}\left(t, \tau_{k}\right) \boldsymbol{M}_{k} \boldsymbol{M}_{k-1} \cdots \boldsymbol{M}_{j} .
$$

One easily verifies that, denoted $\delta_{j}=\tau_{j}-\tau_{j-1}(j=$ $1, \cdots, n)$,

$$
\boldsymbol{U}(t, \tau)=e^{\boldsymbol{A}_{n+1}\left(t-\tau_{n}\right)} \boldsymbol{M}_{n} e^{\boldsymbol{A}_{n} \delta_{n}} \boldsymbol{M}_{n-1} \cdots \boldsymbol{M}_{j} e^{\boldsymbol{A}_{j}\left(\tau_{j}-\tau\right)}
$$

whenever $\tau_{j-1} \leq \tau<\tau_{j} \leq \tau_{j+1} \leq \cdots \leq \tau_{n}<t \leq \tau_{n+1}$.

\section{B. Optimization problem}

Given a positive definite $N \times N$ matrix $\boldsymbol{Q}$, we define the cost functional

$$
\begin{aligned}
F\left(\tau_{1}, \cdots, \tau_{n}\right)= & \int_{0}^{\tau_{1}} \boldsymbol{x}^{T}(t) \boldsymbol{Q} \boldsymbol{x}(t) d t \\
& +\sum_{j=1}^{n}\left(\int_{\tau_{j}}^{\tau_{j+1}} \boldsymbol{x}^{T}(t) \boldsymbol{Q} \boldsymbol{x}(t) d t+h_{j}\left(\tau_{j}\right)\right)
\end{aligned}
$$

where $h_{j}\left(\tau_{j}\right)=H_{j}$ - here $H_{j}$ is a constant - if $\tau_{j}<+\infty$, and $h_{j}\left(\tau_{j}\right)=0$ otherwise. Note that $\tau_{j}<+\infty$ means that the $j$-th switch occurs after a finite amount of time, while $\tau_{j}=$ $+\infty$ means that the $j$-th does not occur, thus its cost is not considered.

Clearly, using the convention $\boldsymbol{U}(+\infty, \tau)=0$ and since

$\int_{\tau_{j-1}}^{\tau_{j}} e^{\boldsymbol{A}_{j}^{T}\left(t-\tau_{j-1}\right)} \boldsymbol{Q} e^{\boldsymbol{A}_{j}\left(t-\tau_{j-1}\right)} d t=\left[e^{\boldsymbol{A}_{j}^{T} t} \boldsymbol{Z}_{j} e^{\boldsymbol{A}_{j} t}\right]_{\tau_{j}-\tau_{j-1}}^{0}$

where $Z_{j}$ are the unique solutions of the Lyapunov equations

$$
\boldsymbol{A}_{j}^{T} \boldsymbol{Z}_{j}+\boldsymbol{Z}_{j} \boldsymbol{A}_{j}=-\boldsymbol{Q},
$$

we get

$$
\begin{aligned}
& F(\left.\tau_{1}, \cdots, \tau_{n}\right) \\
&= \int_{0}^{\tau_{1}} \boldsymbol{x}_{0}^{T} \boldsymbol{U}(t, 0)^{T} \boldsymbol{Q} \boldsymbol{U}(t, 0) \boldsymbol{x}_{0} d t \\
&+\sum_{j=1}^{n}\left[\int_{\tau_{j}}^{\tau_{j+1}} \boldsymbol{x}_{0}^{T} \boldsymbol{U}(t, 0)^{T} \boldsymbol{Q} \boldsymbol{U}(t, 0) \boldsymbol{x}_{0} d t+h_{j}\left(\tau_{j}\right)\right] \\
&= \boldsymbol{x}_{0}^{T} \int_{0}^{\tau_{1}} e^{\boldsymbol{A}_{1}^{T} t} \boldsymbol{Q} e^{\boldsymbol{A}_{1} t} d t \boldsymbol{x}_{0} \\
& \quad+\sum_{j=1}^{n} \boldsymbol{x}_{0}^{T} \boldsymbol{U}\left(\tau_{j}, 0\right)^{T} \boldsymbol{M}_{j}^{T} \\
& \quad \int_{\tau_{j}}^{\tau_{j+1}} e^{\boldsymbol{A}_{j}^{T}\left(t-\tau_{j}\right)} \boldsymbol{Q} e^{\boldsymbol{A}_{j}\left(t-\tau_{j}\right)} d t \boldsymbol{M}_{j} \boldsymbol{U}\left(\tau_{j}, 0\right) \boldsymbol{x}_{0} \\
&= \boldsymbol{x}_{0}^{T} \boldsymbol{Z}_{1} \boldsymbol{x}_{0}+\left(\sum_{j=1}^{n} h_{j}\left(\tau_{j}\right)\right. \\
&\left.\quad+\boldsymbol{x}_{0}^{T} \boldsymbol{U}\left(\tau_{j}, 0\right)^{T}\left[\boldsymbol{M}_{j}^{T} \boldsymbol{Z}_{j+1} \boldsymbol{M}_{j}-\boldsymbol{Z}_{j}\right] \boldsymbol{U}\left(\tau_{j}, 0\right) \boldsymbol{x}_{0}\right) \\
&= \boldsymbol{x}_{0}^{T} \boldsymbol{Z}_{1} \boldsymbol{x}_{0}+\left(\sum_{j=1}^{n} h_{j}\left(\tau_{j}\right)\right. \\
&\left.\quad+\boldsymbol{x}^{T}\left(\tau_{j}^{-}\right)\left[\boldsymbol{M}_{j}^{T} \boldsymbol{Z}_{j+1} \boldsymbol{M}_{j}-\boldsymbol{Z}_{j}\right] \boldsymbol{x}\left(\tau_{j}^{-}\right)\right) .
\end{aligned}
$$




\section{STATE-FEEDBACK CONTROL LAW}

In this section we show that the optimal control law for the optimization problem described in the previous section takes the form of a state-feedback, i.e., to determine if a switch from $\boldsymbol{A}_{j}$ to $\boldsymbol{A}_{j+1}$ should occur it is only necessary to look at the current system state $\boldsymbol{x}$. Thus, the optimization problem can be solved computing a set of state space regions $\mathcal{C}_{j, n}$ : if the system dynamics is $A_{j}$ we will switch as soon as the state reaches a point in the region $\mathcal{C}_{j, n}$, for $j=1, \ldots, n$.

This is an important result because it is well now that a statefeedback control law has many advantages over an open-loop control, including the fact that the computation of the control law can be done off-line as opposed to being performed online. On-line computations are burdensome, especially if a disturbance acting on the system may cause the system state to deviate from its expected value.

To prove this result, we also show constructively how the regions $\mathcal{C}_{j, n}$ can be computed. We first show how the region $\mathcal{C}_{n, n}$ for the last switch can be determined. Secondly we show how inductively the region $\mathcal{C}_{j, n}$ can be computed if the region $\mathcal{C}_{j+1, n}$ is known.

\section{A. Computation of the region for the last switch}

Let us assume that after $n-1$ switches the current system dynamics is that corresponding to matrix $\boldsymbol{A}_{n}$ and the current state vector is $\boldsymbol{y}$ with $\|\boldsymbol{y}\|=1$.

The optimal remaining cost starting from $y$ will consist of two terms: a term due to the time-driven evolution, plus (if the $n$-th switch occurs) the switching cost $H_{n}$.

If no switch occurs and the system evolves with dynamics $\boldsymbol{A}_{n}$ the remaining cost starting from $\boldsymbol{y}$ is only due to the time-driven evolution and is

$$
T_{n, 0}^{*}(\boldsymbol{y})=\boldsymbol{y}^{T} \boldsymbol{Z}_{n} \boldsymbol{y}
$$

where the fist subscript $n$ denotes the current dynamics, and the second subscript 0 denotes the fact that no more switch occurs. We also define, with a notation that will be clarified in the sequel,

$$
\varrho_{n, 0}(\boldsymbol{y})=+\infty
$$

If the system evolves with dynamics $\boldsymbol{A}_{n}$ for a time $\varrho$ and then a switch to $\boldsymbol{A}_{n+1}$ occurs, the remaining cost starting from $\boldsymbol{y}$ only due to the time-driven evolution (disregarding the switching cost) is

$$
\begin{aligned}
T_{n, 1}(\boldsymbol{y}, \varrho)= & \boldsymbol{y}^{T} \boldsymbol{Z}_{n} \boldsymbol{y} \\
& +\boldsymbol{y}^{T} e^{\boldsymbol{A}_{n}^{T} \varrho\left[\boldsymbol{M}_{n}^{T} \boldsymbol{Z}_{n+1} \boldsymbol{M}_{n}-\boldsymbol{Z}_{n}\right] e^{\boldsymbol{A}_{n} \varrho} \boldsymbol{y}}
\end{aligned}
$$

where the fist subscript $n$ denotes the current dynamics, and the second subscript 1 denotes the fact that we allow up to 1 more switches.

Let us denote the value of $\varrho$ that minimize (7) as

$$
\varrho_{n, 1}(\boldsymbol{y})=\arg \min _{\varrho} T_{n, 1}(\boldsymbol{y}, \varrho)
$$

and denote the minimum of (7) as

$$
T_{n, 1}^{*}(\boldsymbol{y})=T_{n, 1}\left(\boldsymbol{y}, \varrho_{n, 1}(\boldsymbol{y})\right) .
$$

Three cases may occur, as shown in Figure 1.

Cases a) and b) are such that $\varrho_{n, 1}(\boldsymbol{y})<+\infty$ i.e., we can obtain an optimal discount on the cost of the time-driven evolution switching after $\varrho_{n, 1}(\boldsymbol{y})$ and the optimal discount is

$$
\gamma_{n, 1}(\boldsymbol{y})=T_{n, 0}^{*}(\boldsymbol{y})-T_{n, 1}^{*}(\boldsymbol{y}) .
$$

Case c) is such that $\varrho_{n, 1}(\boldsymbol{y})=+\infty$ and thus

$$
T_{n, 0}^{*}(\boldsymbol{y})=T_{n, 1}^{*}(\boldsymbol{y})
$$

i.e., there is no advantage in executing the $n$-th switch.

Let us define

$$
E_{n, 0}=0, \quad \text { and } \quad E_{n, 1}=H_{n} .
$$

Taking also into account the switching cost, the optimal remaining cost starting from $\boldsymbol{y}$ is

$$
F_{n}(\boldsymbol{y})=\min _{k=0,1}\left\{T_{n, k}^{*}(\boldsymbol{y})+E_{n, k}\right\} .
$$

and the optimal remaining number of switches is

$$
k_{n}(\boldsymbol{y})=\arg \min _{k=0,1}\left\{T_{n, k}^{*}(\boldsymbol{y})+E_{n, k}\right\} .
$$

Thus the optimal switch should occur after a delay

$$
\delta_{n}(\boldsymbol{y})=\varrho_{n, k_{n}(\mathbf{y})}(\boldsymbol{y}) .
$$

Let us now consider any other vector $\boldsymbol{x}$ such that $\boldsymbol{x}=\lambda \boldsymbol{y}$, with $\lambda \in \mathbb{R}$. We can compute for this new vector the equivalent of (6) and (7), i.e.,

$$
T_{n, 0}^{*}(\boldsymbol{x})=\boldsymbol{x}^{T} \boldsymbol{Z}_{n} \boldsymbol{x}=\lambda^{2} T_{n, 0}^{*}(\boldsymbol{y})
$$

and

$$
\begin{aligned}
T_{n, 1}(\boldsymbol{x}, \varrho)= & \boldsymbol{x}^{T} \boldsymbol{Z}_{n} \boldsymbol{x} \\
& +\boldsymbol{x}^{T} e^{\boldsymbol{A}_{n}^{T} \varrho}\left[\boldsymbol{M}_{n}^{T} \boldsymbol{Z}_{n+1} \boldsymbol{M}_{n}-\boldsymbol{Z}_{n}\right] e^{\boldsymbol{A}_{n} \varrho} \boldsymbol{x} \\
= & \lambda^{2} T_{n, 1}(\boldsymbol{y}, \varrho),
\end{aligned}
$$

Equation (13) is minimized by

$$
\varrho_{n, 1}(\boldsymbol{x})=\arg \min _{\varrho} T_{n, 1}(\boldsymbol{x}, \varrho)=\varrho_{n, 1}(\boldsymbol{y}),
$$

and its minimal value is

$T_{n, 1}^{*}(\boldsymbol{x})=T_{n, 1}\left(\boldsymbol{x}, \varrho_{n, 1}(\boldsymbol{x})\right)=T_{n, 1}\left(\boldsymbol{x}, \varrho_{n, 1}(\boldsymbol{y})\right)=\lambda^{2} T_{n, 1}^{*}(\boldsymbol{y})$.

If we also take into account the switching cost, the optimal remaining cost starting from $\boldsymbol{x}$ is

$$
\begin{aligned}
F_{n}(\boldsymbol{x}) & =\min _{k=0,1}\left\{T_{n, k}^{*}(\boldsymbol{x})+E_{n, k}\right\} \\
& =\min _{k=0,1}\left\{\lambda^{2} T_{n, k}^{*}(\boldsymbol{y})+E_{n, k}\right\},
\end{aligned}
$$




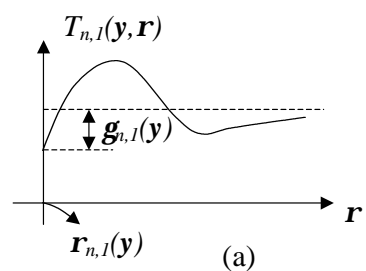

(a)

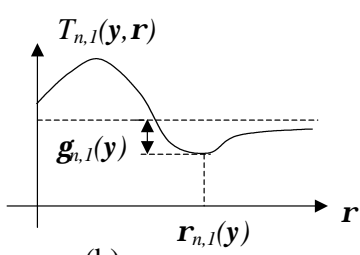

(b)

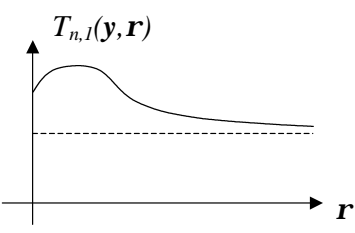

(c)

Fig. 1. Different cases of $T_{n, 1}(\boldsymbol{y}, \varrho)$.

the optimal remaining number of switches starting from $\boldsymbol{x}$ is

$$
\begin{aligned}
k_{n}(\boldsymbol{x}) & =\arg \min _{k=0,1}\left\{T_{n, k}^{*}(\boldsymbol{x})+E_{n, k}\right\} \\
& =\arg \min _{k=0,1}\left\{\lambda^{2} T_{n, k}^{*}(\boldsymbol{y})+E_{n, k}\right\}
\end{aligned}
$$

and the optimal $n$-th switch should occur after a delay

$$
\delta_{n}(\boldsymbol{x})=\varrho_{n, k_{n}(\mathbf{x})}(\boldsymbol{x}) \in\left\{\varrho_{n, k}(\boldsymbol{y}) \mid k=0,1\right\} .
$$

Finally, we can say that a vector $\boldsymbol{x}=\lambda \boldsymbol{y}$ belongs to $\mathcal{C}_{n, n}$ if and only if $\delta_{n}(\boldsymbol{x})=0$, because in this case the optimal remaining cost can be obtained switching as soon as we reach $\boldsymbol{x}$ with no delay. According to the previous discussion, this occurs if and only if $\varrho_{n, 1}(\boldsymbol{y})=0$ (i.e., $T_{n, 1}(\boldsymbol{y}, \varrho)$ has the shape shown in Figure 1.a) and $|\lambda| \geq\left(H_{n} / \gamma_{n, 1}(\boldsymbol{y})\right)^{0.5}$.

Note that to compute the switching region $\mathcal{C}_{n, n}$ and to determine the optimal remaining cost $F_{n}(\boldsymbol{x})$, we only need to compute the value $\varrho_{n, 1}(\boldsymbol{y})$ with a one-parameter optimization (see equations (7) and (8)) for all $\boldsymbol{y}$ on the unitary semi-sphere. The corresponding values of $T_{n, 0}^{*}(\boldsymbol{y})$ and $T_{n, 1}^{*}(\boldsymbol{y})$ can be obtained applying equations (6) and (9), while to determine if a vector $\boldsymbol{x}=\lambda \boldsymbol{y}$ belongs to $\mathcal{C}_{n, n}$ and to compute the corresponding optimal remaining cost we only need to apply equations (16), (17) and (18).

\section{B. Computation of the regions for the intermediate switches}

We now generalize the previous approach to determine the switching regions $\mathcal{C}_{j, n}$, for $j=1, \ldots n-1$.

Assume that:

- we have already computed region $\mathcal{C}_{j+1, n}$;

- for each vector $\boldsymbol{y}$ on the unitary semi-sphere we know the optimal cost $T_{j+1, k}^{*}(\boldsymbol{y})$ for the remaining time-driven evolution that starts from $\boldsymbol{y}$ with dynamics $\boldsymbol{A}_{j+1}$ and allows $k$ more switches (with $k=0, \ldots, n-j$ );

- for all $k=0, \ldots, n-j$ it holds $T_{j+1, k}^{*}(\lambda \boldsymbol{y})=\lambda^{2} T_{j+1, k}(\boldsymbol{y})$.

Let us assume that after $j-1$ switches the current system dynamics is that corresponding to matrix $\boldsymbol{A}_{j}$ and the current state vector is $\boldsymbol{x}=\lambda \boldsymbol{y}$ with $\|\boldsymbol{y}\|=1$.

If no switch occurs and the system evolves with dynamics $\boldsymbol{A}_{j}$ the remaining cost starting from $\boldsymbol{x}=\lambda \boldsymbol{y}$ is

$$
T_{j, 0}^{*}(\boldsymbol{x})=\boldsymbol{x}^{T} \boldsymbol{Z}_{j} \boldsymbol{x}=\lambda^{2} \boldsymbol{y}^{T} \boldsymbol{Z}_{j} \boldsymbol{y}=\lambda^{2} T_{j, 0}^{*}(\boldsymbol{y}) .
$$

We also define

$$
\varrho_{j, 0}(\boldsymbol{y})=+\infty
$$

If the system evolves with dynamics $\boldsymbol{A}_{j}$ for a time $\varrho$, then a switch to $\boldsymbol{A}_{j+1}$ occurs, and then the future evolution is such that only $k-1 \leq n-j$ additional switches occurs, the optimal remaining cost starting from $\boldsymbol{x}$ due to the time-driven evolution (disregarding the switching costs) is

$$
\begin{aligned}
& T_{j, k}(\lambda \boldsymbol{y}, \varrho) \\
& \quad=\boldsymbol{x}^{T}\left[\boldsymbol{Z}_{j}-e^{\boldsymbol{A}_{j}^{T} \varrho} \boldsymbol{Z}_{j} e^{\boldsymbol{A}_{j} \varrho}\right] \boldsymbol{x}+T_{j+1, k-1}\left(\boldsymbol{M}_{j} e^{\boldsymbol{A}_{j} \varrho} \boldsymbol{x}\right) \\
& \quad=\lambda^{2} \boldsymbol{y}^{T}\left[\boldsymbol{Z}_{j}-e^{\boldsymbol{A}_{j}^{T} \varrho} \boldsymbol{Z}_{j} e^{\boldsymbol{A}_{j} \varrho}\right] \boldsymbol{y}+\lambda^{2} T_{j+1, k-1}\left(\boldsymbol{M}_{j} e^{\boldsymbol{A}_{j} \varrho} \boldsymbol{y}\right) \\
& \quad=\lambda^{2} T_{j, k}(\boldsymbol{y}, \varrho),
\end{aligned}
$$

for all $k=1, \ldots, n-j+1$.

Thus for all $\boldsymbol{y}$ on the unitary semi-sphere we compute, solving $n-j+1$ one-parameter optimization problems, the value of $\varrho$ that minimize (20) with $\lambda=1$ for all values of $k=1, \ldots, n-$ $j+1$ :

$$
\varrho_{j, k}(\boldsymbol{y})=\arg \min _{\varrho} T_{j, k}(\boldsymbol{y}, \varrho),
$$

and denote the corresponding minimum as

$$
T_{j, k}^{*}(\boldsymbol{y})=T_{j, k}\left(\boldsymbol{y}, \varrho_{j, k}(\boldsymbol{y})\right) .
$$

Let us define

$$
E_{j, 0}=0
$$

and for $k=1, \ldots, n-j+1$,

$$
E_{j, k}=\sum_{i=1}^{k} H_{j+i-1} .
$$

Taking also into account the switching cost, the optimal remaining cost starting from $\boldsymbol{x}$ is

$$
F_{j}(\boldsymbol{x})=\min _{k=0, \ldots, n-j+1}\left\{\lambda^{2} T_{j, k}^{*}(\boldsymbol{y})+E_{j, k}\right\},
$$

the optimal remaining number of switches is

$$
k_{j}(\boldsymbol{x})=\arg \min _{k=0, \ldots, n-j+1}\left\{\lambda^{2} T_{j, k}^{*}(\boldsymbol{y})+E_{j, k}\right\}
$$

and the optimal switch should occur after a delay

$$
\delta_{j}(\boldsymbol{x})=\varrho_{j, k_{j}(\mathbf{x})}(\boldsymbol{x}) \in\left\{\varrho_{j, k}(\boldsymbol{y}) \mid k=0, \cdots, n-j+1\right\} .
$$

Finally, we can say that a vector $\boldsymbol{x}=\lambda \boldsymbol{y}$ belongs to $\mathcal{C}_{j, n}$ if and only if $\delta_{j}(\boldsymbol{x})=0$. 


\section{Structure of the switching regions}

We now discuss the form that the switching regions may take. Let us first state a trivial fact.

Fact 1: For all $\boldsymbol{x} \in \mathbb{R}^{\mathbb{N}}, j=1, \ldots, n$ and $k=0, \ldots, n-j$ holds

$$
T_{j, k+1}^{*}(\boldsymbol{x}) \leq T_{j, k}^{*}(\boldsymbol{x})
$$

Proof: This can be easily shown by induction on $j$. The result is true for $j=n$ and $k=0,1$, by equations (12) and (13) (base step). Assume the result holds for $j+1$; then it also holds for $j$ given equations (19) and (20) (induction step).

Let us now consider the case of null switching costs.

Proposition 2: Consider the case in which $H_{j}=0$ for all $j=1, \ldots, n$. Then for all $j=1, \ldots, n$ and all $\lambda \in \mathbb{R}$ :

$$
\boldsymbol{y} \in \mathcal{C}_{j, n} \Longrightarrow \lambda \boldsymbol{y} \in \mathcal{C}_{j, n}
$$

i.e., the regions $\mathcal{C}_{j, n}$ are homogeneous.

Proof: Thanks to Fact 1, it is immediate to see that if all costs are null $k_{j}(\boldsymbol{x})=k_{j}(\boldsymbol{y})=n-j+1$ and $\delta_{j}(\boldsymbol{x})=\delta_{j}(\boldsymbol{y})$. Thus $\delta_{j}(\boldsymbol{y})=0 \Longrightarrow \delta_{j}(\boldsymbol{x})=0$.

In the cases of non-null switching costs, the value of $k_{j}(\lambda \boldsymbol{y})$ and correspondingly of $\delta_{j}(\lambda \boldsymbol{y})$ may depend on $\lambda$. However, for any $\boldsymbol{y}$ on the unitary semi-sphere we can define

$$
\tilde{k}_{j}(\boldsymbol{y})=\min \left\{k \mid T_{j, k}^{*}(\boldsymbol{y})=T_{j, n-j+1}^{*}(\boldsymbol{y})\right\} .
$$

It is immediate to see that there exists a $\tilde{\lambda}_{j}(\boldsymbol{y}) \geq 0$ such that

$$
k_{j}(\lambda \boldsymbol{y})=\tilde{k}_{j}(\boldsymbol{y})
$$

for all $\lambda \in \mathbb{R}$ with $|\lambda| \geq \tilde{\lambda}_{j}(\boldsymbol{y})$.

We can thus state the following result.

Proposition 3: For all $j=1, \ldots, n$, and all $\lambda \in \mathbb{R}$ with $|\lambda| \geq$ $\tilde{\lambda}_{j}(\boldsymbol{y})$ :

$$
\tilde{\lambda}_{j}(\boldsymbol{y}) \boldsymbol{y} \in \mathcal{C}_{j, n} \Longrightarrow \lambda \boldsymbol{y} \in \mathcal{C}_{j, n}
$$

\section{NUMERICAL SIMULATIONS}

In this section we present the results of some numerical simulations. In particular, we consider a second order system whose dynamics may only switch between two matrices $\boldsymbol{A}^{(1)}$ and $\boldsymbol{A}^{(2)}$. We also assume that only three switches are possible $(n=3)$ and the initial system dynamics is $\boldsymbol{A}^{(1)}$. Thus, the sequence of switching is $\boldsymbol{A}^{(1)} \rightarrow \boldsymbol{A}^{(2)} \rightarrow \boldsymbol{A}^{(1)} \rightarrow \boldsymbol{A}^{(2)}$, where

$$
\boldsymbol{A}^{(1)}=\left[\begin{array}{cc}
-1 & 1 \\
-18 & -5
\end{array}\right], \quad \boldsymbol{A}^{(2)}=\left[\begin{array}{cc}
1 & -5 \\
1 & -3
\end{array}\right] \text {. }
$$

Note that $\boldsymbol{A}^{(1)}$ and $\boldsymbol{A}^{(2)}$ are stable non-commuting matrices, i.e., $\boldsymbol{A}^{(1)} \boldsymbol{A}^{(2)} \neq \boldsymbol{A}^{(2)} \boldsymbol{A}^{(1)}$. We also assume that all $\boldsymbol{M}_{j}$ are equal to the identity matrix.

We consider two different cases. We firstly assume that no cost is associated to switches. Secondly, we associate a constant cost to each switch.
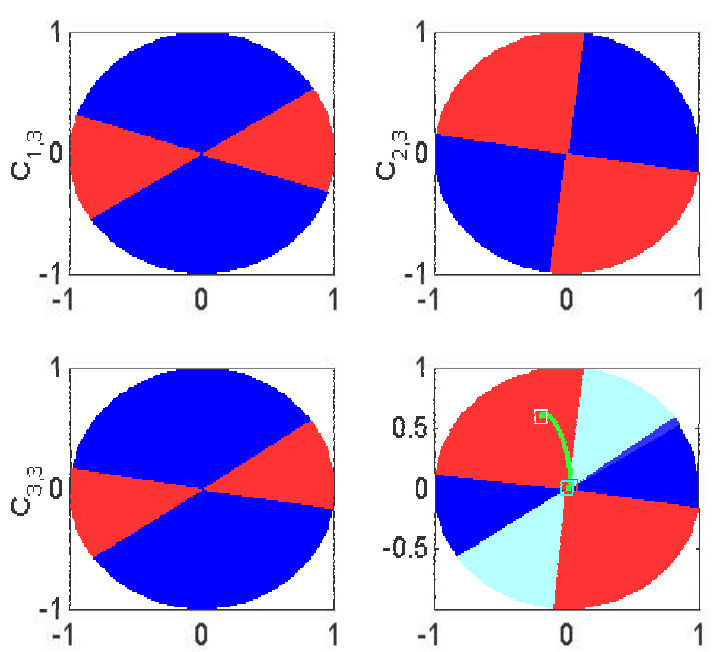

Fig. 2. The switching regions $\mathcal{C}_{j, n}, j=1,2,3$ in the case of no cost associated to switches, and the system evolution for $\boldsymbol{x}_{0}=[-0.2,0.6]$.
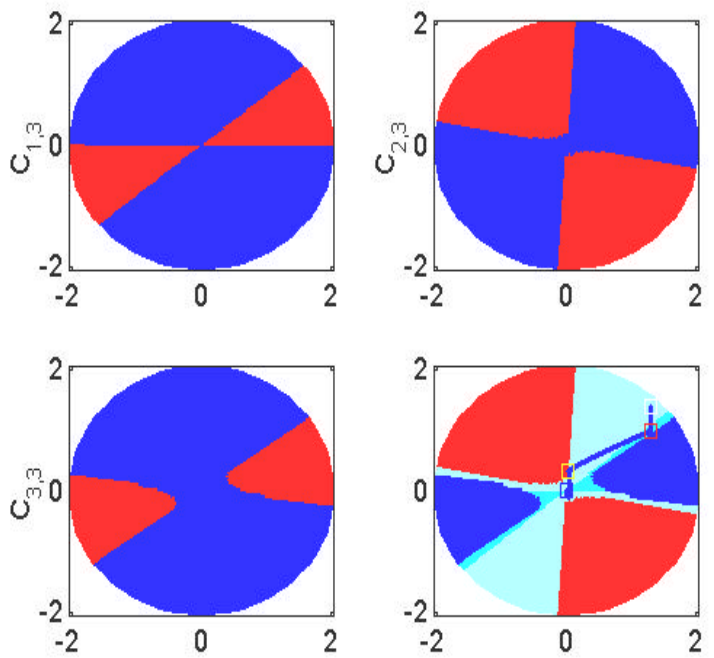

Fig. 3. The switching regions $\mathcal{C}_{j, n}, j=1,2,3$ in the case of non-null costs associated to switches, and the system evolution for $\boldsymbol{x}_{0}=[1.3,1.4]$.

\section{A. First case}

The switching regions $\mathcal{C}_{j, n}, j=1,2,3$, are shown in figure 2 where the following color notation has been used: the lighter region represents the set of states where the system switches to the next dynamics, while the darker region represents the set of states where the system still evolves with the same dynamics.

In the bottom right of figure 2 we have shown the system evolution in the case of $\boldsymbol{x}_{0}=[-0.2,0.6]$.

The switching times are $\tau_{1}=0.61, \tau_{2}=1.34$ and $\tau_{3}=1.49$, and the optimal cost is $F\left(\tau_{1}, \tau_{2}, \tau_{3}\right)=0.19$.

\section{B. Second case}

Now, let us assume that non-null costs are associated to switches. In particular, let us assume that $H_{1}=H_{3}=0.3$ 


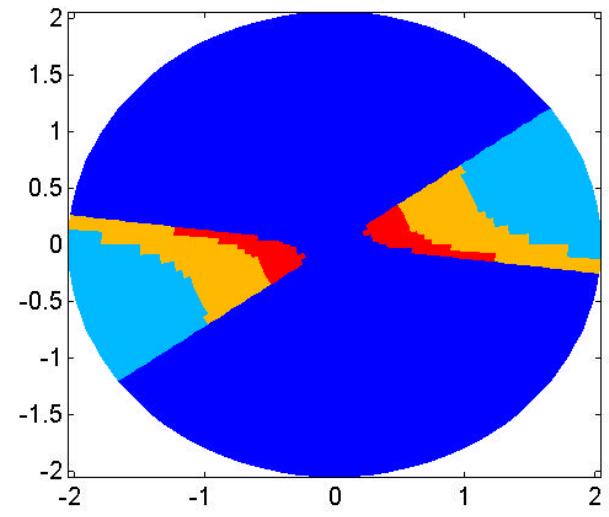

Fig. 4. The switching regions $\mathcal{C}_{3,3}$ for different values of the cost $H_{3} \in$ $\{0.1,0.5,2\}$.

and $H_{2}=0.1$.

The switching regions $\mathcal{C}_{j, n}, j=1,2,3$, are shown in figure 3 where we used the same color notation as above, i.e., the lighter region represents the set of states where the system switches to the next dynamics, and the darker region represents the set of states where the system still evolves with the same dynamics.

In the bottom right of figure 3 we have shown the system evolution in the case of $\boldsymbol{x}_{0}=[1.3,1.4]$. In this case, the switching times are $\tau_{1}=0.014, \tau_{2}=0.5$ and $\tau_{3}=+\infty$, and the optimal cost is $F\left(\tau_{1}, \tau_{2}, \tau_{3}\right)=0.75$.

Let us finally observe that if we assume that the initial state is the same as in the previous case, i.e., $\boldsymbol{x}_{0}=[-0.2,0.6]$, the system evolution is not affected by costs, and is the same as that shown in figure 3.

\section{Modification of the regions}

To show how the switching region $\mathcal{C}_{j, n}$ may change as $H_{j}$ varies, we have also computed for this example the regions $\mathcal{C}_{3,3}$ for different values of $H_{3} \in\{0.1,0.5,2\}$.

These regions are shown in figure 4 , where larger regions correspond to smaller values of $H_{3}$.

\section{Conclusions}

We have considered a special class of switched systems where the switching sequence is finite and pre-assigned, and each subsystem is stable and autonomous.

We showed that the optimal control for this class takes the form of a state feedback, i.e., it is possible to identify a region of the state space such that an optimal switch should occur if and only if the present state belongs to this region. Such a region can be efficiently computed with an off-line numerical procedure.

\section{REFERENCES}

[1] M.S. Branicky, "Stability of Switched and Hybrid Systems", Proc. 33th IEEE Conf. on Decision and Control (Lake Buena Vista, Florida), pp. 34983503, December 1994.

[2] M.S. Branicky, S.K. Mitter, "Algorithms for optimal hybrid control", Proc. 34th IEEE Conf. on Decision and Control, pp. 2661-2666, December 1995.
[3] M.S. Branicky, "Multiple Lyapunov Functions and Other Analysis Tools for Switched and Hybrid Systems", IEEE Trans. on Automatic Control, Vol. 43, pp. 475-482, 1998

[4] M.S. Branicky, R. Hebbar, G. Zhang, "A fast marching algorithm for hybrid systems", Proc. 38th IEEE Conf. on Decision and Control (Phoenix, Arizona), pp. 4897-4902, December 1999.

[5] S. Hedlund, A. Rantzer, "Optimal Control of Hybrid Systems", Proc. 38th IEEE Conf. on Decision and Control (Phoenix, Arizona), pp. 3972-3977, December 1999.

[6] B. Hu, X. Xu, A.N. Michel, P.J. Antsaklis, "Stability Analysis for a Class of Nonlinear Switched Systems", Proc. 38th IEEE Conf. on Decision and Control (Phoenix, Arizona), pp. 4374-4379, December 1999.

[7] P. Riedinger, C. Zanne, F. Kratz "Time Optimal Control of Hybrid Systems", Proc. 1999 American Control Conference (San Diego, CA, USA), June 1999.

[8] P. Riedinger, C. Iung, "Optimal Control for Hybrid Systems: a Hysteresis Example", Proc. 1999 IEEE Conf. on Systems, Man and Cybernetics (Tokyo, Japan), October 1999.

[9] P. Riedinger, F. Kratz, C. Iung, C. Zanne, "Linear Quadratic Optimization for Hybrid Systems", Proc. 38th IEEE Conf. on Decision and Control (Phoenix, Arizona), pp. 3059-3064, December 1999.

[10] X. Xu, P.J. Antsaklis, "Design of stabilizing control laws for second-order switched systems", Proc. 14th IFAC World Congress (Beijing, China), July 1999.

[11] L.Y. Wang, A. Beydoun, J. Cook, J. Sun, I. Kolmanovsky, "Optimal Hybrid Control with Applications to Automative Powertrain Systems", LNCIS 22, Springer-Verlag, 1997. 